\title{
The Conduct of Clinical Trials for Medicinal Products in Europe in the Light of the European Clinical Trials Directive. Review of Regulatory and Practical Aspects in the Different Countries
}

\author{
Claude Dubray ${ }^{1}$, Patricia Maillere ${ }^{2}$, Alain Spriet ${ }^{3}$ and the participants in Round Table $N^{\circ} 1$, Giens XXII \\ 1 Pharmacologie Clinique, CHU Clermont Ferrand, Clermont Ferrand, France \\ 2 IRIS, Groupe Servier, Courbevoie, France \\ 3 Spriet Conseil, Paris, France
}

\section{Keywords:}

ethics committee; clinical trial; pharmacovigilance; Afssaps

\begin{abstract}
The purpose of the clinical trial guidelines is to harmonise legislation in order to ensure consistency within Europe and thereby promote Europe's attractiveness for clinical research while maintaining or improving the protection of subjects who agree to participate.

The French administrative system has hitherto been relatively favourable (with simple notification to a single Ethics Committee per study); it is thus important to maintain competitiveness in these respects, given that, in other areas (the time it takes to establish agreements, recruitment, etc.), other countries have the advantage.

At the moment, this Directive has not been entirely transposed into French law. The pilot period established by the Afssaps (French drug agency) has made it possible to determine very quickly how to set things up.

For Ethics Committees, the situation is more critical in that many points remain that need to be finalised (selection of members, internal rules, Competent Authority/Ethics Committee relationship, etc.). The Giens workshop issued a number of proposals and it hopes, through the Afssaps)/DGS (French agency of health in government)/LEEM Steering Group (French pharmaceutical companies association), to be able to help establish an efficient system which also correctly protect the patient.
\end{abstract}

\section{Introduction}

Every European country has organised its own, independent legislation governing clinical trials. Some countries required prior authorisation from the relevant health authorities before any trial whereas others only required such authorisation for studies on patients, or just required notification. Some countries had established an agreement process based on a single Ethics Committee per study whereas others required one Committee for each Investigating Centre. The information to be submitted varied from country to country as did requirements updates and information during conduct of the trial.

\footnotetext{
^ For a list of participants, please see the end of the article.
}

In the 1980's, the Commission proposed harmonising the legislation in order to ensure consistency within Europe and thereby promote Europe's attractiveness for clinical research while maintaining or improving the protection of subjects who agree to participate.

The final version of the European Directive was published on November 28, 2001 and transposition will soon be finalized in the various different countries. At the time of the Round Table studies, several Application Decrees were still lacking, notably in the area of how Ethics Committees should operate.

Various inquiries, notably conducted by the LEEM have shown that, in the past, the French administration was relatively lenient in that notification rather than authorisation was required 
for studies, and because the Huriet Law had allowed to set up a simple system with one Ethics Committee authorisation per study.

The goal was to compare the French situation with those in the other major European countries, and to formulate proposals as to how to maintain or improve its attractiveness.

Parallel discussions were conducted in five sub-groups:

- review of the situation;

- ethics Committees;

- financial conditions;

- pharmacovigilance;

- clinical trial registries.

\section{Review of the situation}

This sub-group was presided over by Christine Marey and produced tables to compare how the Directive is being transposed in ten countries, namely The Czech Republic, Denmark, Finland, France, Germany, Italy, Norway, Poland, Spain and Sweden, on the basis of existing texts without taking any other regulatory provisions or cultural mores into account. Stock was however taken of the experience of the sub-group's members.

Many subjects were examined: the roles and responsibilities of the qualified authorities and Ethics Committees, composition, administrative procedures, approval of sites, registries, compensation, etc.

These tables can be consulted on the Giens Web site. ${ }^{[1]}$

Concretely, during the Afssaps pilot phase,when reviews by the Ethics Committees are still governed by the Huriet Law, France maintains competitive time frames for trial approval. The average time-to inclusion of the first patient is, on average, 110115 days, the key delay often stemming from the need to negotiate a contract with the hospital.

The group's first recommendation is therefore addressed to hospital directors who are asked to agree to start to prepare contracts without waiting for the approval of the Ethics Committee (as is still often the case).

In terms of time frames, the Afssaps has taken a competitive position by including the validation period in the 60 -day time frame, without providing for a clock stopin the event of question. If the company cannot reply in the stipulated period, the file must be re-submitted. The group's second recommendation is that, in these circumstances, the fees should not have to be paid again in order to avoid wasting time. With respect to the transparency of the deadline, it should be noted that the Afssaps specifically mentions the D60 date in its acknowledgement of receipt. The efforts already made to make in-house evaluators more accessible to everyone concerned for any clarification requested by the sponsors are to be encouraged. This is so in major countries (United Kingdom, Sweden...) and really speeds up the last part of the procedure.

Some ideas originating in other countries could be applied in France, e.g.: (i) call procedures as in the United Kingdom for both the competent authority and the Ethics Committee; (ii) the idea of co-sponsorship: responsibilities for handling the study file, conducting the trial and managing pharmacovigilance could be shared around between different entities; (iii) agreement with comments (as practiced in the United Kingdom, Germany, Sweden, Finland and Poland).

Special attention was paid to the provision of investigational medicinal products because this is an important impediment to academic research projects. Currently, there is five times more non-commercial research per inhabitant in the United States than in Europe. There is a provision in a draft European Guideline for the pharmaceutical manufacturer to provide help without being the trial sponsor. If this is to become a reality, it is important that the company not be re-qualified as the sponsor, should any problem other than one related to product quality be encountered.

Some European countries including France foresee that health care systems may provide for this in different ways according each country's system. In France, medicinal products in clinical trials can be reimbursed by the Social Security System as long as the product is being used in an indication for which it is licensed for reimbursement, or if the Ministry decides, after consulting UNCAM (Union Nationale des Caisses d'Assurance Maladie) and HAS (Haute Autorité de Santé) on the basis of a public heath interest criteria.

It is important that this opportunity exists. However, the global duration of this procedure remains an obstacle.

A quicker solution could come from the text being debated at the moment in Parliament which provides for inclusion of the medicinal products in studies of routine medical care. This subject should be monitored closely.

\section{Ethics committees}

This second has been presided by Philippe Rusch.

The European Directive provides for a single Ethics Committee approval per study. In reality, in many countries, approval is often required from the Committee of each clinical center $(e . g$. the UK) or region (Germany and Spain) involved. Therefore, in terms of the simplicity of the procedure, France is once again in a relatively good position.

With respect to how examine what, two structures - the Afssaps and the Ethics Committee - evaluate the trial's methodology; there is therefore the possibility of disagreement. This 
situation should be reviewed. It is clear that the Afssaps naturally has a more global vision since it evaluates all protocols related to a given product or products in the same class. The members of Ethics Committees present felt that it was always useful to provide an independent evaluation of the methodology in the application if one is available, e.g. European Scientific Advice.

With respect to transposition of the Directive, many Decrees are still lacking and not all Ethics Committees have been reappointed.

The strengths of the French system were pointed up: (i) the number of Ethics Committees will be the same as previously; (ii) inter-regionalism should speed up file handling; (iii) 10-40\% of members still need to be identified; (iv) how old files and their amendments are dealt with should be defined in a pragmatic way; (v) the legality of advises should be thoroughly assessed: they are now binding whereas before they were purely consultative. It is therefore necessary to establish a format to check the validity of given advises (quorum, etc.); (vi) the Decree on collections is eagerly awaited. Several proposals have been advanced: approval by default, definition of the end of research according to the protocol in a pragmatic manner, work on collection and change of end purpose.

Many topics require clarification to ensure that the performance of Ethics Committees is not affected: functioning, Afssaps/Ethics Committee dialogue and internal rules.

A number of recommendations were issued to preserve the competitive edge at Ethics Committees level:

- France has chosen to include representatives of patient associations in Ethics Committees. These positions have been fulfilled in almost all Committees. The training of these members is a process that needs consolidation. It should be noted that 160 members have been trained by Eurordis-Inserm. Training to evaluation will be a subject for every new member;

- the Decree of April 2006 requires the systematic presence of an expert in biostatistics. In practice, this point proves problematic and the validity of Ethics Committee approvals is compromised. It is therefore proposed to modify the Decree in order to replace the word biostatistician by a competent person in methodology.

The subject of harmonising the rulings of European Ethics Committees was brought up, especially in the framework of the EUREC network (European Network of Research Ethics Committees) ${ }^{[2]}$ which gathers Committees in the following countries: United Kingdom, Danemark, Deutschland, Spain, Italy, France, Switzerland, Finland, Lithuania and Slovakia.

The first topic addressed by this group was description of the existing instruments, operating procedures and definition of a useful training body for all countries.
Major differences soon stood out:

- for example, certain committees prioritise the methodological evaluation of the trial whereas others tend to focus on the protection of participating subjects;

- some seek to train their members to the level of professional evaluators whereas others wish to preserve the naivety of representatives of civil society.

This dialogue, difficult as it may be, deserves encouragement.

\section{Registries}

The third sub-group presided over by Claire Sibenaler specifically addressed the subject of registries insofar as the Afssaps plans to set up such a registry.

Many registries exist already:

Firstly, to enhance the transparency of the design of trials, the main editors have asked that, in order to be published, every trial be listed at a site independent of the sponsor. In fact, only two sites are currently accredited. ${ }^{[3,4]}$

The pharmaceutical companies said that they would make the protocols and results of their trials available on-line, often at their own sites. A few discussion points on timing remain: proof of concept studies and a few secondary criteria which might bring confidential items for future development. The FIIM portal (IFPMA International Federation of Pharmaceutical Manufacturers \& Associations) ${ }^{[5]}$ exists and facilitates requests; the WHO is also establishing another portal. Institutional sites are planned: Europharm is to make available on-line information about current products on the market (Summary of Product Characteristics, Package Leaflets, etc.) and give notice of ongoing study protocols.

There is a consensus that the multiplication of such data bases is undesirable because it will be very difficult to keep them up to date, thus avoiding contradictory information.

In France, the Afssaps database already exists for certain diseases (rare diseases, hepatitis) and the INCA wishes to establish one for cancer trials.

A few European agencies wish, like the Afssaps, to establish an on-line site about clinical trials (Spain, Italy). The special value of the Afssaps site is that it provides information in French for French patients. It has been proposed not to transfer EUDRACT fields automatically (for reasons of understanding) nor disseminate information still considered confidential by the sponsor (be it a pharmaceutical company or an academic institution).

\section{Pharmacovigilance reports}

The fourth sub-group presided over by Véronique Lamarque addressed Pharmacovigilance reports. 
The basic subject was the amount of information communicated to comply with regulations, and what is really useful to remember and assess. The competent authorities in the various countries are already used to receiving spontaneous reports during trials, this is new for the Ethics Committees which must now define their assignment with respect to this information: how to handle and evaluate it. .

The following recommendations were proposed:

- generalised utilisation of the CIOMS (Council for Intenational Organizations Of Medical Sciences) form, clear reference to the trial, information restricted to SUSARs (SUspected Severe Adverse Reactions) and taking into account events predefined in the protocol;

- regular summary reports with clear, well-formed conclusions;

- reconsideration of the obligation to send reports of individual cases to Ethics Committees at the European level;

- to study the possibility of defining a European supervisor responsible for handling PV for a structure in the case of cosponsorship;

- to pay great attention to the text on Vigilance on NonExperimental Medicinal Products which is currently being drafted.

\section{Evaluation fees}

The last sub-group presided over by Elisabeth Rouffiac focussed on differences in costs and compensation in different countries.

Evaluation fees charged by the competent authorities or Ethics Committees (application file, inspections, evaluation of annual reports, etc.) are not currently key factors in the choice of country. Very high fees are charged by some Committees in countries other than France; this point needs to be monitored.

The compensation of researchers poses special problems in France. This parameter is traditionally evaluated by the Ethics Committee outside France and does not incite much debate. In France, the sums are evaluated by the French medical association CNO (Conseil National de l'Ordre) which can be a worry when it comes to the time it takes to set a trial up. The dialogue between sponsors and $\mathrm{CNO}$ about this topic should be pursued.

Compensating patients is now possible in France as in other countries (the UK, Germany, Spain). Only the constraints involved may be compensated according to guidelines and sums previously approved by the Ethics Committee.

\section{Conclusion}

The purpose of the clinical trial guidelines is to harmonise legislation in order to ensure consistency within Europe and thereby promote Europe's attractiveness for clinical research while maintaining or improving the protection of subjects who agree to participate. The French administrative system has hitherto been relatively favourable (with simple notification to a single Ethics Committee per study); it is thus important to maintain competitiveness in these respects, given that, in other areas (the time it takes to establish agreements, recruitment, etc.), other countries have the advantage.

At the moment, this Directive has not been entirely transposed into French law. The pilot period established by the Afssaps have made it possible to determine very quickly how to set things up.

For Ethics Committees, the situation is more critical in that many points remain that need to be finalised (selection of members, internal rules, Competent Authority/Ethics Committee relationship, etc.). The Giens workshop issued a number of proposals and it hopes, through the Afssaps/DGS/LEEM Steering Group, to be able to help establish an efficient system which also correctly protect the patient.

\section{Participants}

Michel Abitboul (Quintiles), Béatrice Barraud (INSERMDAPS), Marie Castera-Tellier (CHU, Lille), François Chapuis (CHU, Lyon), François Faurisson (Eurordis), Elizabeth FrijaOrvoen (Présidente CPP Hotel Dieu, Paris), Jean Genève (FNCLCC), Anne-Sophie Ginon (MCF en droit Université Paris X, Nanterre), Corinne Guérin (Groupe Hospitalier Cochin), Thierry Herguetta (Président CPP Salpétrière, Paris), Denis Lacombe (EORTC), Véronique Lamarque (Pfizer), Christian Libersa (Pharmacologie, Faculté de Médecine, Lille), Brigitte Marchenay (Roche), Christine Marey (Servier), Annie Métro (ANRS), Armelle Mijonnet (Merck Sharp \& Dohme Chibret), Claire Orefice (Cythéris), Gérard Pons (Hôpital St Vincent de Paul, Paris), Nathalie Quideau (Sanofi), Isabelle Richard-Lordereau (Glaxo Smith Kline), Christian Rollin (Bristol Myers Squibb), Elisabeth Rouffiac (Abbott France/Pharmacie), Philippe Rusch (Président CPPRB, Saint-Etienne), Claire Sibenaler (LEEM), Sandra Touratier (Afssaps, Unité Essais Cliniques).

\section{References}

1. http://wwW.ateliersdegiens.org

2. http://www . eurecnet.org

3. http://www.clinicaltrials.gov

4. http://www. controlled-trials.com

5. http://www.ifpma.org

Correspondence and offprints: Patricia Maillere, IRIS, Groupe Servier, 6 place des Pléiades, 92415 Courbevoie Cedex, France.

E-mail: maillere@fr.netgrs.com 